\title{
Multi-Histogram Equalization Methods for Contrast Enhancement and Brightness Preserving
}

\author{
David Menotti, Laurent Najman, Jacques Facon, and Arnaldo de A. Araújo
}

\begin{abstract}
Histogram equalization (HE) has proved to be a simple and effective image contrast enhancement technique. However, it tends to change the mean brightness of the image to the middle level of the gray-level range, which is not desirable in the case of images from consumer electronics products. In the latter case, preserving the input brightness of the image is required to avoid the generation of non-existing artifacts in the output image. To surmount this drawback, BiHE methods for brightness preserving and contrast enhancement have been proposed. Although these methods preserve the input brightness on the output image with a significant contrast enhancement, they may produce images with do not look as natural as the input ones. In order to overcome this drawback, this work proposes a novel technique called Multi-HE, which consists of decomposing the input image into several sub-images, and then applying the classical HE process to each one. This methodology performs a less intensive image contrast enhancement, in a way that the output image presents a more natural look. We propose two discrepancy functions for image decomposing, conceiving two new Multi-HE methods. A cost function is also used for automatically deciding in how many sub-images the input image will be decomposed on. Experiments show that our methods preserve more the brightness and produce more natural looking images than the other HE methods. ${ }^{\text {. }}$
\end{abstract}

Index Terms - Contrast enhancement, brightness preserving, histogram equalization, multi-threshold selection.

\section{INTRODUCTION}

The histogram of a discrete gray-level image represents the frequency of occurrence of all gray-levels in the image [1]. Histogram equalization (HE) is a technique commonly used for image contrast enhancement, since $\mathrm{HE}$ is computationally fast and simple to implement. It works by flattening the histogram and stretching the dynamic range of the gray-levels by using the cumulative density function of the image.

\footnotetext{
1 This work was supported in part by the CNPq/MCT and CAPESCOFECUB/MEC, Brazilian Government's research support agencies.

D. Menotti the correspondence author and Laurent Najman are with the Laboratoire Algorithmique et Architecture des Systèmes Informatiques (A2SI), Université Paris-Est, Groupe ESIEE-Paris Noisy-le-Grand, BP99, 93162, France (e-mail: d.menotti@esiee.fr, 1.najman@esiee.fr).

J. Facon is with the Programa de Pós-Graduação em Informática Aplicada, Pontifícia Universidade Católica do Paraná, Prado Velho, Curitiba, 80215901, Brazil (e-mail: facon@ppgia.pucpr.br).

A. de A. Araújo and D. Menotti (as well) are with the Núcleo de Processamento Digital de Imagens, Departamento de Ciência da Computação, Universidade Federal de Minas Gerais, Pampulha, Belo Horizonte, 31270010, Brazil (e-mail: arnaldo@dcc.ufmg.br and menotti@dcc.ufmg.br).
}

Contributed Paper

Manuscript received July 15, 2007

$00983063 / 07 / \$ 20.00$ C 2007 IEEE
Despite its success for image contrast enhancement, this technique has a well-known drawback: it does not preserve the brightness of the input image on the output one. This drawback makes the use of HE not suitable for image contrast enhancement on consumer electronic products, such as video surveillance, where preserving the input brightness is essential to avoid the generation of non-existing artifacts in the output image. To overcome such drawback, variations of the classic $\mathrm{HE}$ technique have proposed to first decompose the input image into two sub-images, and then perform $\mathrm{HE}$ independently in each sub-image. These methods, described in details in Section III, use some statistical measures - which consider the value of the graylevels in the image, during the decomposition step. Another method, which will not be described in Section III but is of relevance for this work, is the variational framework based on histogram transformation for image contrast enhancement and brightness preserving with maximum entropy (BPHEME) presented in [2]. Although in [2] the authors claim that their method is a HE one, we claim that it is actually a histogram specification of an entropy distribution. Both the methods based on Bi-HE and the method proposed in [2] perform image contrast enhancement with success while preserving the input brightness in some extend, but they might generate images with do not look as natural as the input ones. Such result is unacceptable for consumer electronics products.

In order to enhance contrast, preserve brightness and produce natural looking images, this article proposes a MultiHE (MHE) technique which first decomposes the input image into several sub-images, and then applies the classical HE process to each of them. We present two discrepancy functions to decompose the image, conceiving two MHE methods for image contrast enhancement, i.e., Minimum Within-Class Variance MHE (MWCVMHE) and Minimum Middle Level Squared Error MHE (MMLSEMHE). A cost function, taking into account both the discrepancy between the input and enhanced images and the number of decomposed sub-images, is used to automatically make the decision of in how many sub-images the input image will be decomposed on. The remaining of this work is organized as follows. As the proposed method use many concepts previously introduced in the literature, Section II presents some basic definitions regarding gray-level images, whereas Section III describes previous works. The proposed methods are introduced in Section IV. Results of our methods are presented, discussed and compared with other HE methods in Section V. Finally, conclusions are drawn in Section VI. 


\section{BASIC DEFINITIONS}

In this section, we present some basic definitions for graylevel images, which will be used throughout this work.

Let $¥$ and $\varnothing$ denote the set of natural and integer numbers, respectively. Let $X^{m n}$ be a subset of points $(x, y) \in ¥^{2}$, such that $0 \leq<m$, and $0 \leq y<n$, where $m$ and $n$ denote the dimensions of $X^{m n}$. Note that $\left\|X^{m n}\right\|=m \times n$, where $\|Y\|$ denote the cardinality of the set $Y$. A mapping $I$, from $X^{m n}$ to $\varnothing_{L}=\{0, \ldots, L-1\}$, is called an image. In applications, $L$ is typically 256 . For a point $(x, y) \in X^{m n}, l=I(x, y)$ is called the level of the point $(x, y)$ in $I$.

Let $l_{s}$ and $l_{f}$ be levels of the image $I$, where $0 \leq l_{s} \leq l_{f}<L$. Let $I\left[l_{s}, l_{f}\right] \subseteq I$ be composed by all mappings from points $(x, y) \in X^{m n}$ to $\left\{l_{s}, l_{s}+1, \ldots, l_{f}-1, l_{f}\right\}$. The sub-mapping $I\left[l_{s}, l_{f}\right]$ defines a (sub-)image of $I$.

The definition above was presented to facilitate the definition of a sub-histogram and its probability functions, which are necessary for the definition of Bi- and Multi-HE methods. In the followings, when the boundaries $\left[l_{s}, l_{f}\right]$ of the image $I$ are omitted, they are assumed to be $[0, L-1]$.

Let $X_{l}^{m n}$ be a subset of $X^{m n}$, such that for all $(x, y) \in X_{l}^{m n} \subseteq X^{m n}$, we have $I(x, y)=l$. Let $H_{l}^{I}$ be the absolute frequency of the level $l$ in the image $I$, where $0 \leq l \leq L-1$, i.e., $H_{l}^{I}=\left\|X_{l}^{m n}\right\|$.

The mapping $H^{I}$ from the levels of the image $I$ to their absolute frequency levels, i.e., $H^{I}: \phi_{L} \rightarrow ¥$, is called the histogram of the image $I$. Note that $H_{l}^{I}=H_{l}^{I\left[l_{s}, l_{f}\right]}$, with $0 \leq l_{s} \leq l \leq l_{f}<L$.

Let $P_{l}^{I\left[l_{s}, l_{f}\right]}$ be the relative frequency (or the probability) of level $l$ in the (sub-)image $I\left[l_{s}, l_{f}\right]$, i.e.,

$$
P_{l}^{I\left[l_{s}, l_{f}\right]}=H_{l}^{I} / \sum_{i=l_{s}}^{l_{f}} H_{i}^{I},
$$

where $0 \leq l_{s} \leq l \leq l_{f} \leq L-1$. The function $P^{I\left[l_{s}, l_{f}\right]}$, which is composed by all $P_{l}^{I\left[l_{s}, l_{f}\right]}$, is the probability density function of the image $I\left[l_{s}, l_{f}\right]$.

Let $C_{l}^{I\left[l_{s}, l_{f}\right]}$ be the probability distribution (or the cumulative probability density) of the level $l$ in the image $I\left[l_{s}, l_{f}\right]$, i.e.,

$$
C_{l}^{I\left[l_{s}, l_{f}\right]}=\sum_{i=l_{s}}^{l_{f}} P_{i}^{I\left[l_{s}, l_{f}\right]},
$$

where $0 \leq l_{s} \leq l \leq l_{f}<L$. The function $C^{I\left[l_{s}, l_{f}\right]}$ composed by all
$C_{l}^{I\left[l_{s}, l_{f}\right]}$ is the probability distribution function (or the cumulative probability density function) of the image $I\left[l_{s}, l_{f}\right]$.

Let $I\left[l_{s}, l_{f}\right]$ be a sub-image of $I$. We define the mean (or the brightness) of the image $I\left[l_{s}, l_{f}\right]$ as

$$
l_{m}\left(I\left[l_{s}, l_{f}\right]\right)=\sum_{l=l_{s}}^{l_{f}} l \times P_{l}^{I\left[l_{s}, l_{f}\right]} .
$$

Let $I\left[l_{s}, l_{f}\right]$ be a sub-image of $I$. We define the standard deviation (or the contrast) of the image $I\left[l_{s}, l_{f}\right]$ as

$$
l_{\sigma}\left(I\left[l_{s}, l_{f}\right]\right)=\sqrt{\sum_{l=l_{s}}^{l_{f}}\left(l-l_{m}\left(I\left[l_{s}, l_{f}\right]\right)\right)^{2} \times P_{l}^{I\left[l_{s}, l_{f}\right]}} .
$$

\section{Previous Work}

This section describes some previous works in the literature which make use of the HE method with the purpose of brightness preserving. We start by describing the classical HE (CHE) method in Section III.A. The CHE method was the base for the other four methods, namely BBHE, DSIHE, MMBEBHE and RMSHE, which will be later described in this section. Notice that these four extensions of the CHE method have one main point in common: they decompose the input image into two or more sub-images, and then equalize the histograms of these sub-images independently. In contrast, the major difference among these methods is the criteria they use to decompose the input image into two or more subimages. The first method, described in Section III.B, divides the input image into two by using its mean gray-level. An extension of this method, which recursively segments the input image, is later described in Section III.E. Section III.C presents a method which uses the equal area value to segment the images, whereas the method described in Section III.D segments images by taking into account the level which yields the minimum brightness error between the input and the enhanced images. To conclude, Section III.F presents some final remarks.

Note that, from now on, $I$ and $O$ denote the input (or the original) and the output (or the processed) images, respectively.

\section{A. Classical HE Method (CHE)}

This section describes the CHE method for gray-level images in detail, since this method is the core of this work. The goal of HE method is to uniformly distribute the histogram of an image over the entire range of gray-levels, increasing the image contrast.

Let $H^{I\left[l_{s}, l_{f}\right]}, P^{I\left[l_{s}, l_{f}\right]}$ and $C^{I\left[l_{s}, l_{f}\right]}$ be defined as in Section II. Let $H^{O\left[l_{s}, l_{f}\right]}$ be the uniform histogram of the output image, where any level $l$, with $l_{s} \leq l \leq l_{f}$, has the same amount of pixels, or the same density, i.e.,

$$
P_{l}^{O\left[l_{s}, l_{f}\right]}=\frac{1}{l_{f}-l_{s}+1},
$$


The cumulative density function $C^{O\left[l_{s}, l_{f}\right]}$ is defined in function of $l$ as

$$
C_{l}^{O\left[l_{s}, l_{f}\right]}=\sum_{i=l_{s}}^{l_{f}} P_{i}^{I\left[l_{s}, l_{f}\right]}=\frac{i-l_{s}+1}{\left(l_{f}-l_{s}+1\right)},
$$

The $l^{\prime}$ ' output level corresponding to the input level $l$ is obtained as the one that minimizes the difference between $C_{l^{\prime}}^{O\left[l_{s}, l_{f}\right]}$ and $C_{l}^{O\left[l_{s}, l_{f}\right]}$. In other words, the output level $l^{\prime}$ for the input level $l$ can be computed as the transformation function $T^{I\left[l_{s}, l_{f}\right]}(l)$, i.e.,

$$
l^{\prime}=T^{I\left[l_{s}, l_{f}\right]}(l)=l_{s}+\left\|\left(l_{f}-l_{s}\right) \times C_{l}^{I\left[l_{s}, l_{f}\right]}\right\|,
$$

where $\|z\|$ stands for the nearest integer to $z \in \square$.

To generate the enhanced output image $O\left[l_{s}, l_{f}\right]$ using this transformation, for any pixel $l=I\left[l_{s}, l_{f}\right](x, y)$, we obtain its respective output level $O\left[l_{s}, l_{f}\right](x, y)$ as $l^{\prime}=T^{I\left[l_{s}, l_{f}\right]}(l)$.

The high performance of the HE in enhancing the contrast of an image is a consequence of the dynamic range expansion of the gray-level's image domain. That is, theoretically the output image enhanced by a HE method uses all the graylevel's image domain, i.e., from 0 up to $L-1$. Besides, the CHE tries to produce an output image with a flatten histogram, i.e., a uniform distribution. Based on information theory, the entropy of a message source will get the maximum value when the message respects the uniform distribution property [4]. This means that an image enhanced by the $\mathrm{CHE}$ method has the maximum information (i.e., the entropy) with respect to its original one. However, the CHE method barely satisfies the uniform distribution property in images with discrete gray-level domains.

Despite of the advantages offered the CHE method, it can introduce a significant change in the image brightness, i.e., its mean gray-level. That is, thanks to the uniform distribution specification of the output histogram, the CHE method shifts the brightness of the output image to the middle gray-level, i.e., $L / 2$. This change in brightness is not desirable when applying the CHE scheme into consumer electronics devices, for instance TV and video surveillance. This is because it may introduce unnecessary visual deterioration to the output image.

\section{B. Brightness Bi-HE Method (BBHE)}

In order to overcome the drawback introduced by the $\mathrm{CHE}$ method described in the previous subsection, a brightness preserving $\mathrm{Bi}-\mathrm{HE}$ (BBHE) method was proposed in [5]. The essence of the BBHE method is to decompose the original image into two sub-images, by using the image mean graylevel, and then apply the CHE method on each of the subimages. In [5], it is mathematically shown that the BBHE method produces an output image with the value of brightness (the mean gray-level) located in the middle of the mean of the input image and the middle gray-level (i.e., $L / 2$ ).

\section{Dualistic Sub-Image HE Method (DSIHE}

Following the same basic ideas used by the BBHE method of decomposing the original image into two sub-images and then equalize the histograms of the sub-images separately, [4] proposed the so called equal area dualistic sub-image HE (DSIHE) method. Instead of decomposing the image based on its mean gray level, the DSIHE method decomposes the images aiming at the maximization of the Shannon's entropy [6] of the output image. For such aim, the input image is decomposed into two sub-images, being one dark and one bright, respecting the equal area property (i.e., the sub-images has the same amount of pixels).

In [4], it is shown that the brightness of the output image $O$ produced by the DSIHE method is the average of the equal area level of the image $I$ and the middle gray level of the image, i.e., $L / 2$. The authors of [4] claim that the brightness of the output image generated by the DSIHE method does not present a significant shift in relation to the brightness of the input image, especially for the large area of the image with the same gray-levels (represented by small areas in histograms with great concentration of gray-levels), e.g., images with small objects regarding to great darker or brighter backgrounds.

\section{D.Minimum Mean Brightness Error Bi-HE Method (MMBEBHE)}

Still following the basic principle of the BBHE and DSIHE methods of decomposing an image and then applying the CHE method to equalize the resulting sub-images independently, [3] proposed the minimum mean brightness error Bi-HE (MMBEBHE) method. The main difference between the BBHE and DSIHE methods and the MMBEBHE one is that the latter searches for a threshold level $l_{t}$ that decomposes the image $I$ into two sub-images $I\left[0, l_{t}\right]$ and $I\left[l_{t}+1, L-1\right]$, such that the minimum brightness difference between the input image and the output image is achieved, whereas the former methods consider only the input image to perform the decomposition.

Once the input image is decomposed by the threshold level $l_{t}$, each of the two sub-images $I\left[0, l_{t}\right]$ and $I\left[l_{t}+1, L-1\right]$ has its histogram equalized by the classical HE process, generating the output image. Assumptions and manipulations for finding the threshold level $l_{t}$ in $O(L)$ time complexity were made in [3]. Such strategy allows us to obtain the brightness $l_{m}(O[O, l] \cup O[l+1, L-1])$ of the output image without generating the output image for each candidate threshold level $l$, and its aim is to produce a method suitable for real-time applications.

\section{E. Recursive Mean-Separate HE Method (RMSHE)}

Recall that the extensions of the CHE method described so far in this section were characterized by decomposing the original image into two new sub-images. However, an extended version of the BBHE method (see Section III.B) 
proposed in [7], and named recursive mean-separate HE (RMSHE), proposes the following. Instead of decomposing the image only once, the RMSHE method proposes to perform image decomposition recursively, up to a scale $r$, generating $2^{r}$ sub-images. After, each one of these sub-images $I^{r}\left[l_{s}, l_{f}\right]$ is independently enhanced using the CHE method.

Note that when $r=0$ (no sub-images are generated) and $r=1$, the RMSHE method is equivalent to the CHE and BBHE methods, respectively. In [7], they mathematically showed that the brightness of the output image is better preserved as $r$ increases. Note that, computationally speaking, this method presents a drawback: the number of decomposed sub-histograms is a power of two.

\section{F. An Insight on the Results Produced by HE Methods}

The previous sections described methods which use HE with the purposed of preserving the brightness of gray-level images. Fig. 1 shows, for the girl image, the output images produced by these HE methods. In turn, Table I shows the values of the brightness and contrast obtained for these images.

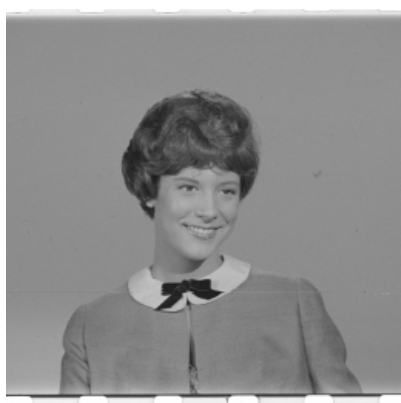

Original



BBHE

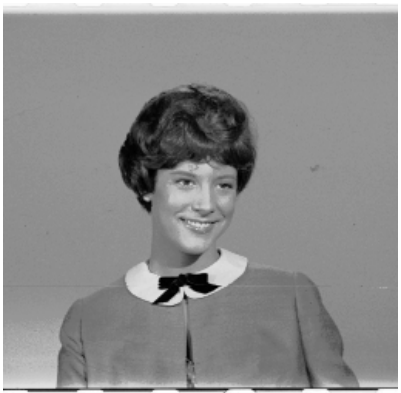

$\operatorname{RMSHE}(r=2)$

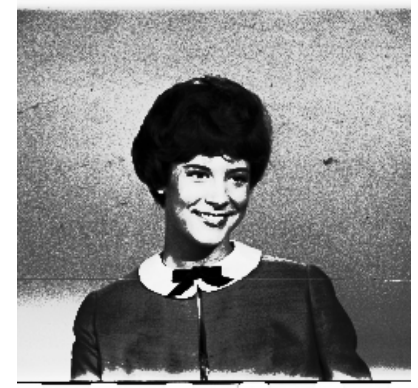

HE

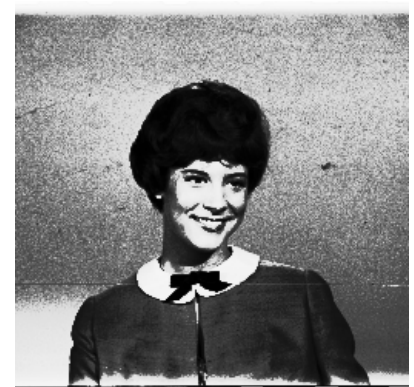

DSIHE

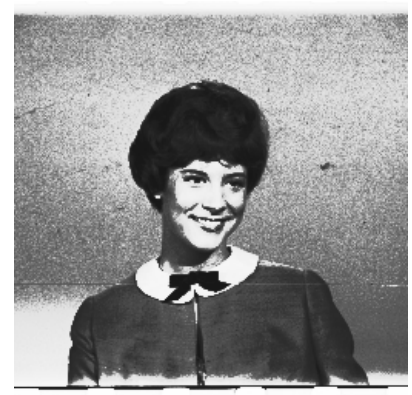

MMBHEBE
Fig. 1. An example of image contrast enhancement and brightness preserving on the girl image.
By analyzing the data in Table I and the images in Fig. 1, we observe that the only method which preserves the brightness of the input image and generates a natural looking image is the RMSHE method ( $r=2)$. Recall that this method is based on multi-histogram decomposition or, in other words, on the recursive decomposition of the image into two subimages. Still looking at the data presented in Table I, we can also conclude that the Bi-HE methods are not robust regarding brightness preserving. To overcome this drawback, Section IV introduces two new robust methods for image contrast enhancement and brightness preserving, also capable of producing natural looking images.

TABLE I

Brightness Preserving Methods fro Image Contrast ENHANCEMENT.

\begin{tabular}{lcc}
\hline \hline \multicolumn{1}{c}{ Method } & Brightness & Contrast \\
\hline Original & 139.20 & 29.70 \\
HE & 133.94 & 75.47 \\
BBHE & 162.78 & 70.09 \\
DSIHE & 131.66 & 75.42 \\
RMSHE $(r=2)$ & 139.77 & 37.81 \\
MMBEBHE & 144.97 & 68.70 \\
\hline
\end{tabular}

\section{Multi-Histogram Equalization Methods for Contrast Enhancement and Brightness Preserving}

As mentioned before, the HE method enhances the contrast of an image but cannot preserve its brightness (which is shifted to the middle gray-level value). As a result, the HE method can generate unnatural and non-existing objects in the processed image. In contrast, Bi-HE methods can produce a significant image contrast enhancement and, at some extend, preserve the brightness of the image. However, the generated images might not have a natural appearance. To surmount such drawbacks, the main idea of our proposed methods is to decompose the image into several sub-images, such that the image contrast enhancement provided by the HE in each subimage is less intense, leading the output image to have a more natural look. The conception of such methods arises two questions.

The first question is how to decompose the input image. As $\mathrm{HE}$ is the focus of the work, the image decomposition process is based on the histogram of the image. The histogram is divided into classes, determined by threshold levels, where each histogram class represents a sub-image. The decomposition process can be seen as an image segmentation process executed through multi-threshold selection [8]. The second question is in how many sub-images an image should be decomposed on. This number depends on how the image is decomposed, and so this question is directly linked with the first question.

In order to answer these questions, Section IV.A presents two functions to decompose an image based on threshold levels, whereas the algorithm used to find the optimal threshold levels is presented in Section IV.B. Finally, a 
criterion for automatically selecting the number of decomposed sub-images is exposed in Section IV.C.

\section{A. Multi-Histogram Decomposition}

Many HE-based methods have been proposed in the literature to decompose an image into sub-images by using the value of some statistical measure based on the image's graylevel value [3]-[5], [7]. These methods aim to optimize the entropy or preserve the brightness of the image. Here, we will focus our attention on decomposing an image such that the enhanced images still have a natural appearance. For such aim, we propose to cluster the histogram of the image in classes, where each class corresponds to a sub-image. By doing that, we want to minimize the brightness shift yielded by the $\mathrm{HE}$ process into each sub-image. With the minimization of this shift, this method is expected to preserve both the brightness and the natural appearance of the processed image.

From the multi-threshold selection literature point of view, the problem stated above can be seen as the minimization of the within-histogram class variance [8], where the within-class variance is the total squared error of each histogram class with respect to its mean value (i.e., the brightness). That is, the decomposition aim is to find the optimal threshold set $T^{k}=\left\{t_{1}^{k}, \ldots, t_{k-1}^{k}\right\}$ which minimizes the decomposition error of the histogram of the image into $k$ histogram classes and decomposes the image $I[0, L-1]$ into $k$ sub-images $I\left[l_{s}^{1, k}, l_{f}^{1, k}\right], \ldots, I\left[l_{s}^{k, k}, l_{f}^{k, k}\right]$, where $l_{s}^{j, k}$ and $l_{f}^{j, k}$ stand for the lower and upper gray-level boundaries of each sub-image $j$ when the image is decomposed into $k$ sub-images. They are defined as: $l_{s}^{j, k}=t_{j-1}^{k}$, if $j>1$, and $l_{s}^{j, k}=0$ otherwise, and $l_{f}^{j, k}=t_{j}^{k}+1$, if $j \neq k$, and $l_{f}^{j, k}=L-1$ otherwise. The discrepancy function for decomposing the original image into $k$ sub-images following the minimization of within-class variance can be expressed as

$$
\operatorname{Disc}(k)=\sum_{j=1}^{k} \sum_{l=l_{s}^{j, k}}^{l_{f}^{j, k}}\left(l-l_{m}\left(I\left[l_{s}^{j, k}, l_{f}^{j, k}\right]\right)\right)^{2} P_{l}^{I[0, L-1]} .
$$

The method conceived with this discrepancy function will be called Minimum Within-Class Variance MHE (MWCVMHE). Note that the mean gray-level (i.e., the brightness) of each sub-image processed by the CHE method is theoretically shifted to the middle gray-level of its range, i.e., $l_{m}\left(O\left[l_{s}, l_{f}\right]\right)=l_{m m}\left(I\left[l_{s}, l_{f}\right]\right)=\left(l_{s}+l_{f}\right) / 2$. As we want to minimize the brightness shift of each processed sub-image, such that the global processed image has its contrast enhanced and its brightness preserved (creating a natural looking output image), we focus our attention on the brightness of the output image. Hence, instead of using the mean $l_{m}\left(I\left[l_{s}, l_{f}\right]\right)$ of each input sub-image $I\left[l_{s}, l_{f}\right]$ in the discrepancy function, we propose to use its middle level $\left(l_{s}+l_{f}\right) / 2$, since every enhanced sub-image $O\left[l_{s}, l_{f}\right]$ will theoretically have its mean value (brightness) on the middle level of the image range thanks to the specification of a uniform histogram distribution. Therefore, a new discrepancy function is proposed and it is expressed as

$$
\operatorname{Disc}(k)=\sum_{j=1}^{k} \sum_{l=l_{s}^{j, k}}^{l_{f}^{j, k}}\left(l-l_{m m}\left(I\left[l_{s}^{j, k}, l_{f}^{j, k}\right]\right)\right)^{2} P_{l}^{I[0, L-1]},
$$

where $l_{m m}\left(I\left[l_{s}^{j, k}, l_{f}^{j, k}\right]\right)$ stands for the middle value of the image $I\left[l_{s}^{j, k}, l_{f}^{j, k}\right]$. The method conceived with this discrepancy function will be called Minimum Middle Level Squared Error MHE (MMLSEMHE).

Algorithm 1: Computing $\operatorname{Disc}(k)$ and $P T(k, L-1)$.

Data: $\varphi(p, q)$ - discrepancy of sub-image $I(p, q)$

Result: $D(p)_{q}$ - disc. function $\operatorname{Disc}(p)$ up to level $q$

Result: $P T$ - optimum thresholds matrix

1 for $q \leftarrow 0 ; q<L ; q++$ do $D(l)_{q} \leftarrow \varphi(0, q)$;

2 for $p \leftarrow 1 ; p \leq k ; p++$ do

$3 \quad D(p+1)_{p} \leftarrow D(p)_{p-1}+\varphi(p-1, p-1)$;

$4 \quad P T(p+1, p) \leftarrow p-1$;

5 for $q \leftarrow p+1 ; q \leq L-k+p ; q++$ do

$6 \quad D(p+1)_{q} \leftarrow-\infty ;$

7 for $l \leftarrow p-1 ; l \leq q-1 ; l++$ do

$8 \quad$ if $\left(D(p+1)_{q}>D(p)_{l}+\varphi(l+1, q)\right)$ then

$9 \quad D(p+1)_{q} \leftarrow D(p)_{l}+\varphi(l+1, q)$;

$10 \quad P T(p+1, q) \leftarrow l$;

\section{B. Finding the Optimal Thresholds}

The task of finding the optimal $k-1$ threshold levels which segment an image into $k$ classes can be easily performed by a dynamic programming algorithm with $O\left(k L^{2}\right)$ time complexity [9]. Algorithm 1 presents this algorithm, where $\varphi(p, q)$ stands for the "discrepancy contribution" of the subimage $I[p, q]$, i.e.,

$$
\varphi(p, q)=\sum_{l=p}^{q}(l-\gamma)^{2} P_{l}^{I[0, L-1]},
$$

and $\gamma$ stands for $l_{m}(I[p, q])$ or $l_{m m}(I[p, q])$, depending on the discrepancy function used (see (8) and (9)).

Once Algorithm 1 is run, the optimal threshold vector $T^{k}$ can be obtained through a back-searching procedure on $P T$, i.e.,

$$
t_{k}^{j}=P T\left(j+1, t_{j+1}^{k^{*}}\right)
$$


where $1 \leq j<k, \quad t_{j+1}^{k^{*}}=L-1$ if $j+1=k$, and $t_{j+1}^{k^{*}}=t_{j+1}^{k}$ otherwise.

\section{C.Automatic Thresholding Criterion}

This section presents an approach to automatically choose in how many sub-image the original image should be decomposed on. This decision is a key point of our work, which has three main aims: 1) contrast enhancement; 2) brightness preserving; 3) natural appearance. Nonetheless, these goals cannot be all maximize simultaneously. We take into account that as the number of sub-images in which the original image is decomposed increases, the chance of preserving the image brightness and natural appearance also increases. However, the chances of enhancing the image contrast decrease. To decide on how many sub-images the original image should be decomposed, this tradeoff should be considered. Hence, we propose to use a cost function, initially used in [10], to automatically select the number of decomposed sub-images. This cost function takes into account both the discrepancy between the original and processed images (which is our own aim decomposition function) and the number of subimages to which the original image is decomposed, and it is defined as

$$
C(k)=\rho(\operatorname{Disc}(k))^{1 / 2}+\left(\log _{2} k\right)^{2},
$$

where $\rho$ is a positive weighting constant. The number of decomposed sub-images $k$ is automatically given as the one which minimizes the cost function $C(k)$. It is shown in [10] that the cost function presented in (12) has a unique minimum. Hence, instead of finding the value $k$ which minimizes $C(k)$ throughout $k$ values range, it is enough to search for $k$ from 0 up to a value where $C(k)$ starts to increase.

\section{V.RESULTS AND DISCUSSION}

In this section, we report results of experiments comparing our proposed methods with the other HE methods described in Section III and the method proposed in [2]. The input images used in the experiments were the ones previously used in [2]-[5], [7]. They are named as they were in the works where they first appeared: arctic hare, bottle, copter, couple, Einstein, F16, girl, hands, house, jet, U2, woman (girl in [2]). Images were extracted from the CVG-UGR database [11] and provided by the authors of [3], [7].

Table II shows the number of sub-images automatically obtained by the methods MWCVMHE and MMLSEMHE, represented by the columns $l_{m}$ and $l_{m m}$, respectively. These values were obtained using the threshold criterion for image decomposition exposed in Section IV.C, and the weighting constant $\rho$ with the value 0.8 (as done in [10]).

In practice, our methods take less than $50 \mathrm{~ms}$ to find the number $k$, decompose and enhance an image on a Pentium $\mathrm{IV}-2 \mathrm{GHz}$.
To start our analysis, for each image, we computed the brightness (the mean) and the contrast (the standard deviation) of the original and the output images obtained by the $\mathrm{HE}$ methods. Moreover, in order to assess the appropriateness of the processed images for consumer electronics products, we computed the PSNR measure [12] as well. In the image processing literature, the PSNR has been used as a standard measure to evaluate and compare compression and segmentation algorithms [12]. It is well-known that a processed image with good quality (with respect to the original one) presents $P S N R$ values within $30 \mathrm{~dB}$ and $40 \mathrm{~dB}$ [12].

TABLE II

Automatic SElection OF THE NUMber OF SUb-IMAGeS - $k$.

\begin{tabular}{lll||lll}
\hline \hline \multicolumn{1}{c}{ Image } & $\boldsymbol{l}_{\boldsymbol{m}}$ & $\boldsymbol{l}_{\boldsymbol{m} \boldsymbol{m}}$ & \multicolumn{1}{c}{ Image } & $\boldsymbol{l}_{\boldsymbol{m}}$ & $\boldsymbol{l}_{\boldsymbol{m} \boldsymbol{m}}$ \\
\hline arctic hare & 5 & 7 & Girl & 5 & 6 \\
Bottle & 6 & 6 & hands & 5 & 6 \\
Copter & 6 & 6 & house & 6 & 6 \\
Couple & 5 & 6 & jet & 5 & 5 \\
Einstein & 6 & 7 & U2 & 4 & 4 \\
F16 & 5 & 7 & woman & 6 & 7 \\
\hline
\end{tabular}

The values of brightness, contrast and PSNR obtained for each image are presented in Tables III, IV and V, respectively. These tables are divided into three parts: 1) The names and the data values of original images (for the PSNR table, the values of original images are constant, i.e., $\infty)$; 2) The data values obtained by the Uni- and BiHE methods, i.e., HE, BBHE, DSIHE, MMBEBHE, and BPHEME; 3) The values obtained by the MHE methods, i.e., RMSHE ( $r=2)$, and our proposed MWCVMHE and MMLSEMHE.

In Tables III and IV, we first compare the data values (image brightness and image contrast, respectively) of each image in parts 2 (i.e. Uni- and Bi-HE) and 3 (i.e. MHE) of the table with the data values of the input image in part 1. For each image, the best data values in each part of the table appear in gray. In a second step, we compare the best values in parts 2 and 3 of the tables against each other (i.e. Uni- and Bi-HE methods against MHE methods). The best value is dark-grayed, the worse lightgrayed.

Let us first analyze the results in Table III, regarding the brightness of the original and the HE processed images. By observing the absolute difference between the value of brightness in the original and the processed images (i.e., the brightness preservation), we state that: 1) The images produced by our proposed methods are better in preserving the brightness of the original images in 8 out of 12 images; 2) Even thought our methods are not always the best brightness preserving ones, their resulting brightness is always very close to the brightness of the original images; 3) The MMLSEMHE method has shown to be more robust than the MWCVMHE method in terms of brightness preservation. 
TABLE III

\begin{tabular}{|c|c|c|c|c|c|c|c|c|c|}
\hline Image & Original & HE & BBHE & DSIHE & ММВЕВНЕ & BPHEME & $\operatorname{RMSHE}(r=2)$ & MWCVMHE & MMLSEMHE \\
\hline arctic hare & 220.89 & 139.45 & 199.18 & 184.76 & 209.22 & 222.92 & 218.05 & 217.96 & 220.13 \\
\hline bottle & 78.76 & 128.35 & 94.15 & 97.73 & 82.36 & 79.37 & 81.08 & 78.94 & 79.08 \\
\hline copter & 191.44 & 128.69 & 174.23 & 164.47 & 188.57 & 192.12 & 188.43 & 190.2 & 190.53 \\
\hline couple & 33.35 & 129.83 & 66.47 & 77.23 & 49.79 & 33.96 & 43.46 & 36.54 & 35.45 \\
\hline Einstein & 107.75 & 128.83 & 126.99 & 119.78 & 108.84 & 109 & 117.85 & 110.5 & 108.64 \\
\hline F16 & 179.2 & 129.42 & 180.22 & 163.24 & 180.24 & 180.32 & 180.4 & 184.99 & 178.93 \\
\hline girl & 139.2 & 133.94 & 162.78 & 131.66 & 144.97 & 145.21 & 139.77 & 139.46 & 140.05 \\
\hline hands & 27.99 & 179.71 & 52.99 & 46.64 & 46.06 & 38.36 & 31.75 & 41.61 & 31.63 \\
\hline House & 68.97 & 129.86 & 94.00 & 95.48 & 70.48 & 70.74 & 77.01 & 72.68 & 71.35 \\
\hline jet & 201.11 & 129.33 & 196.15 & 174.25 & 201.51 & 201.93 & 200.42 & 200.75 & 201.7 \\
\hline $\mathrm{U} 2$ & 32.51 & 131.33 & 49.32 & 74.21 & 39.78 & 33.56 & 37.06 & 32.66 & 33.55 \\
\hline woman & 113.13 & 128.52 & 129.07 & 124.43 & 114.17 & 114.12 & 113 & 113.84 & 113.24 \\
\hline
\end{tabular}

TABLE IV

IMAGE CONTRAST - STANDARD DEVIATION $\left(\sigma=\sqrt{\sum_{l=0}^{L-1}(l-\mu) \times P(l)}\right)$

\begin{tabular}{|c|c|c|c|c|c|c|c|c|c|}
\hline Image & Original & HE & BBHE & DSIHE & ММВЕВНЕ & BPHEME & $\operatorname{RMSHE}(r=2)$ & MWCVMHE & MMLSEMHE \\
\hline arctic hare & 49.07 & 86.73 & 71.91 & 81.29 & 57.9 & 34.87 & 52.76 & 56.69 & 49.11 \\
\hline bottle & 52.07 & 73.34 & 73.47 & 75.7 & 59.65 & 63.65 & 59.12 & 55.19 & 54.83 \\
\hline copter & 40.66 & 73.9 & 72.7 & 76.76 & 52.52 & 55.89 & 52.05 & 44.85 & 44.48 \\
\hline couple & 31.57 & 71.81 & 74.13 & 79.5 & 48.46 & 32.98 & 53.26 & 35.83 & 32.02 \\
\hline Einstein & 37.11 & 73.56 & 73.87 & 73.92 & 62.33 & 72.14 & 57.92 & 40.05 & 37.59 \\
\hline F16 & 45.12 & 74.57 & 67.68 & 77.4 & 68.79 & 62.61 & 61.08 & 55.3 & 46.74 \\
\hline girl & 29.7 & 75.47 & 70.09 & 75.42 & 68.7 & 74.67 & 37.81 & 35.37 & 31.47 \\
\hline hands & 54.36 & 27.29 & 60.92 & 75.8 & 69.39 & 21.74 & 59.9 & 50.51 & 54.69 \\
\hline house & 38.25 & 73.61 & 75.14 & 75.55 & 55.43 & 59.66 & 56.84 & 41.93 & 39.72 \\
\hline Jet & 52 & 74.31 & 64.71 & 78.33 & 54.36 & 49.7 & 56.79 & 57.19 & 55.92 \\
\hline U2 & 25.62 & 72.23 & 64.58 & 78.39 & 50.00 & 32.14 & 44.68 & 36.52 & 29.63 \\
\hline woman & 49.19 & 73.51 & 73.62 & 73.69 & 66.11 & 72.8 & 62.57 & 52.26 & 50.69 \\
\hline
\end{tabular}

TABLE V

$$
P S N R=10 \times \log _{10}(L-1)^{2} / M S E
$$

\begin{tabular}{l||ccccc||ccc|}
\hline \hline \multicolumn{1}{c||}{ Image } & HE & BBHE & DSIHE & MMBEBHE & BPHEME & RMSHE $(r=2)$ & MWCVMHE & MMLSEMHE \\
\hline arctic hare & 8.11 & 16.63 & 13.09 & 23.55 & 22.95 & 30.74 & 31.44 & 40.27 \\
bottle & 12.88 & 18.68 & 17.53 & 28.44 & 25.72 & 29.68 & 35.99 & 36.71 \\
copter & 10.61 & 15.95 & 14.2 & 25.5 & 23.2 & 25.62 & 33.83 & 34.77 \\
couple & 7.57 & 13.18 & 11.61 & 19.86 & 38.54 & 19.65 & 30.59 & 40.16 \\
Einstein & 15.08 & 15.15 & 15.58 & 18.91 & 16.21 & 19.51 & 31.42 & 34.53 \\
F16 & 11.92 & 20.69 & 16.02 & 20.32 & 21.61 & 22.72 & 24.43 & 37.1 \\
girl & 13.03 & 13.3 & 12.99 & 14.03 & 13.19 & 28 & 29.39 & 33.03 \\
hands & 4.36 & 19.58 & 17.76 & 19.99 & 17.18 & 30.93 & 24.49 & 35.82 \\
house & 10.82 & 14.27 & 14.07 & 21.41 & 19.93 & 21.36 & 31.81 & 36.37 \\
Jet & 9.51 & 22.5 & 14.37 & 30.78 & 23.99 & 27.85 & 29.14 & 31.74 \\
U2 & 6.99 & 15.06 & 10.94 & 19.87 & 27.32 & 22.12 & 26.21 & 31.08 \\
woman & 17.83 & 17.73 & 18.25 & 21.6 & 19.23 & 23.67 & 28.83 & 34.53 \\
\hline
\end{tabular}

We perform a similar analysis to the one performed in Table III in Table IV. By observing the contrast values, we state that: 1) The method DSIHE produces the best image contrast enhancement in 10 out of 12 images, losing only twice for the classical HE method; 2) The RMSHE ( $r=2$ - four sub-images) presents the best image contrast enhancement among the MHE methods in 10 out of 12 images, losing only twice for our
MWCVMHE method; 3) The MMLSEMHE method produces the smaller image contrast enhancement - this is the price to pay when we want to obtain at the same time image contrast enhancement, brightness preserving, and natural looking images. Nonetheless, as will be shown in a further visual analysis of images, the images produced by the MMLSEMHE method are the best ones regarding natural look. 


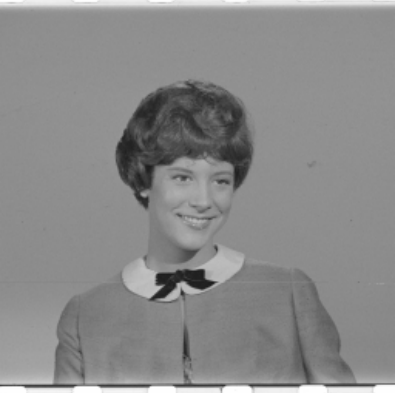

original

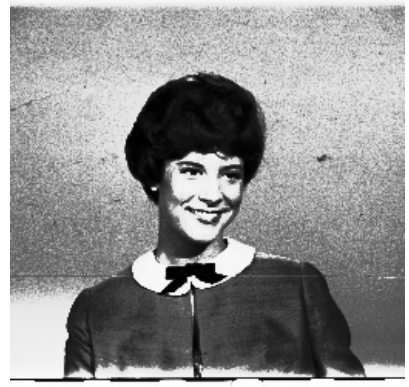

BPHEME

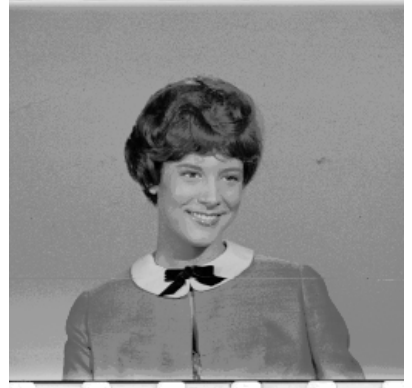

MWCVMHE $(k=5)$

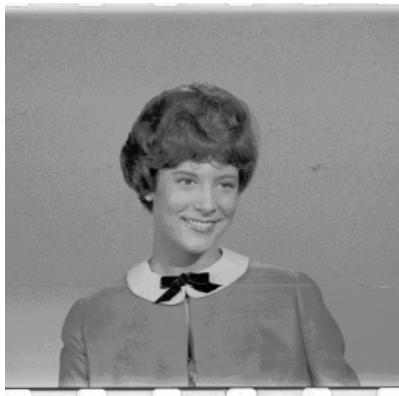

MMLSEMHE $(k=6)$

Fig. 2. Enhancement for the girl image based on BPHEME, MWCVMHE, and MMLSEMHE methods.



original

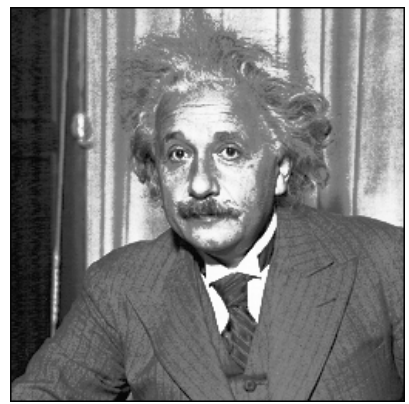

$\operatorname{RMSHE}(r=2)$

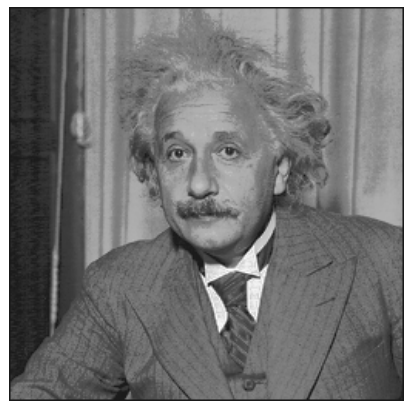

MWCVMHE $(k=6)$

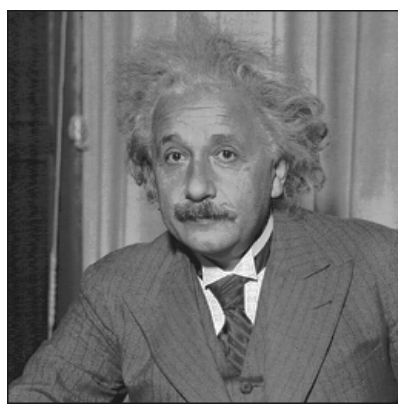

MMLSEMHE $(k=7)$

Fig. 3. Enhancement for the Einstein image based on RMSHE ( $r=2$ ), MWCVMHE, and MMLSEMHE methods.

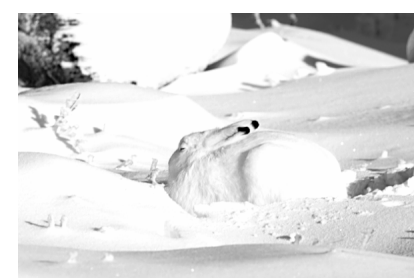

original

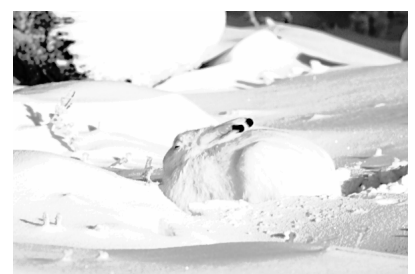

$\operatorname{RMSHE}(r=2)$



MWCVMHE $(k=5)$

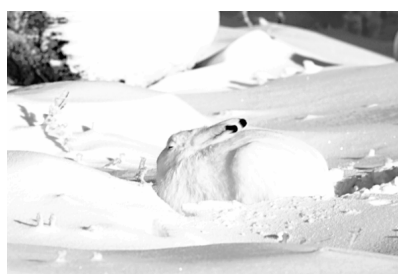

MMLSEMHE $(k=7)$

Fig. 4. Enhancement for the arctic hare image based on RMSHE ( $r=2$ ), MWCVMHE, and MMLSEMHE methods.

Finally, we analyze the data presented in Table V. In Table $\mathrm{V}$, the best values of PSNR are highlighted in gray. Recall that the greater the value of the PSNR, the better it is. Looking at these figures, we observe that the images processed by the MMLSEMHE method produces the best PSNR values, as they are within the range $[30 \mathrm{~dB}, 40 \mathrm{~dB}]$. It is based on this result we argue that the MMLSEMHE method performs image contrast enhancement and brightness preserving and still produce images with a natural looking. Moreover, this result corroborates, in practice, our hypothesis that the MMLSEMHE method, using the discrepancy function in (9), yields images with the best PSNR values among all HE methods.

Once the images were analyzed considering their brightness, contrast and PSNR, we performed an image visual assessment. Remark that all the 12 input images, their histograms, their respective enhanced images and equalized histograms (obtained by all the methods listed in Table III), adding up to more than 200 images, can be seen in [13]. Here we present an analysis of 3 images: girl, Einstein and artic hare. Fig. 2 shows the resulting images obtained by the BPHEME method [4] and our proposed ones for the girl image. Note that the output images obtained by $\mathrm{Bi}-\mathrm{HE}$ and the
RMSHE methods for girl can be observed at Fig. 1. By visually inspecting the images on these two figures, we can clearly see that only the MHE methods (i.e., RMSHE $(r=2)$, MWCVMHE and MMLSEMHE methods) are able to generate natural looking images and still offer contrast enhancement.

Fig. 3 shows the Einstein image and the resulting images obtained by the MHE methods, i.e., RMSHE $(r=2)$, MWCVMHE and MMLSEMHE. By observing the processed images, it is noticeable that our proposed methods are the only ones among the MHE methods that can produce natural looking images. Recall that the other methods are worst than MHE methods for producing natural looking images.

Fig. 4 shows the images obtained by applying the MHE methods to the image artic hare. We chose this picture because it shows that, even thought the image contrast enhancement produced by our methods is sometimes limited, they can enhance particular and interesting parts of an image. Observe that on the upper right corner of the images we can perceive contrast enhancement. Nonetheless, the RMSHE $(r=2)$ and MWCVMHE methods generate better enhancement on that region than the MMLSEMHE method. 
After analyzing the data presented on Tables III-V and visually observing some processed images, we can conclude that: 1) The MMLSEMHE method produces images with better quality than the other methods with respect to the PSNR measure; 2) Nonetheless, a better image contrast enhancement can be obtained by the MWCVMHE method, which also presents satisfactory brightness preserving and natural looking images 3) The RMSHE method $(r=2)$ should be employed if more contrast enhancement than offered by the MMLSEMHE and MWCVMHE methods is desired. However, in this case, the processed image may present some annoying and unnatural artifacts (for instance Fig. 3-RMSHE $(r=2))$.

\section{Conclusion}

In this work, we proposed and tested a new framework called MHE for image contrast enhancement and brightness preserving which generated natural looking images. The experiments showed that our methods is better on preserving the brightness of the processed image (in relation to the original one) and yields images with natural appearance, at the cost of contrast enhancement. The contributions of this work are threefold: 1) An objective comparison among all the HE methods using quantitative measures, such as the PSNR, brightness and contrast; 2) An analysis showing the boundaries of the $\mathrm{HE}$ technique and its variations (i.e., $\mathrm{Bi}$ - and Multi-HE methods) for contrast enhancement, brightness preserving and natural appearance; 3 ) Our proposed methods.

\section{ACKNOWLEDGMENT}

The authors are grateful to G.L. Pappa for reading the first drafts of this paper.

\section{REFERENCES}

[1] R. Gonzalez and R. Woods, Digital Image Processing, $2^{\text {nd }}$ ed. Prentice Hall, Jan. 2002.

[2] C. Wang and Z. Ye, "Brightness preserving histogram equalization with maximum entropy: A variational perspective," IEEE Trans. on Consumer Electronics, vol. 51, no. 4, pp. 1326-1334, Nov. 2005.

[3] S.-D. Chen and A. Ramli, "Minimum mean brightness error bi-histogram equalization in contrast enhancement," IEEE Trans. on Consumer Electronics, vol. 49, no. 4, pp. 1310-1319, Nov. 2003.

[4] Y. Wang, Q. Chen, and B. Zhang, "Image enhancement based on equal area dualistic sub-image histogram equalization method," IEEE Trans. on Consumer Electronics, vol. 45, no. 1, pp. 68-75, Feb. 1999.

[5] Y.-T. Kim, "Contrast enhancement using brightness preserving bihistogram equalization," IEEE Trans. on Consumer Electronics, vol. 43, no. 1, pp. 1-8, Feb. 1997.

[6] C. Shannon, "A mathematical theory of communication," Bell Syst. Tech. J., vol. 27, pp. 379-423, 1948.

[7] S.-D. Chen and A. Ramli, "Contrast enhancement using recursive meanseparate histogram equalization for scalable brightness preservation," IEEE Trans. on Consumer Electronics, vol. 49, no. 4, pp. 1301-1309, Nov. 2003

[8] N. Otsu, "A threshold selection method from grey-level histograms," IEEE Trans. on Systems, Man and Cyb., vol. 9, no. 1, pp. 41-47, 1979.

[9] M. Luessi, M. Eichmann, G. Schuster, and A. Katsaggelos, "New results on efficient optimal multilevel image thresholding," in IEEE International Conference on Image Processing, 2006, pp. 773-776.
[10] J.-C. Yen, F.-J. Chang, and S. Chang, "A new criterion for automatic multilevel thresholding," IEEE Trans. on. Image Processing, vol. 4, no. 3, pp. 370-378, Mar. 1995.

[11] CVG-URG,“database,” 2007, http://decsai.ugr.es/cvg/dbimagenes/.

[12] M. Rabbani and P. Jones, Digital Image Compression Techniques, $1^{\text {st }}$ ed. Bellingham, WA, USA: Society of Photo-Optical Instrumentation Engineers (SPIE), 1991.

[13] D. Menotti, “images," 2007, http://www.dcc.ufmg.br/ menotti/MHE/.

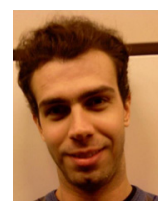

David Menotti was born in Andirá, Paraná, Brazil. He received his Computer Engineering and Informatics Applied Master degrees from Pontifícia Universidade Católica do Paraná, Brazil, in 2001 and 2003, respectively. Since 2004, he is a $\mathrm{PhD}$ student at Universidade Federal de Minas Gerais, Belo Horizonte, Brazil. And in 2005, he also started a Cotutelle $\mathrm{PhD}$ at Université Paris-Est/Groupe ESIEE, Paris, France.

His research interests include image processing, pattern recognition, computer vision and algorithms for information retrieval systems.

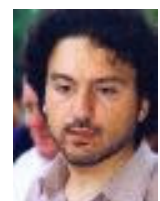

Laurent Najman received the Habilitation à Diriger les Recherches from the Université Paris-Est, Marne-laVallée, France, in 2006, the Ph.D. degree in applied mathematics (Félicitations du Jury) from the Université Paris Dauphine, Paris, France, in 1994, and the Ing. degree from the Ecole des Mines de Paris in 1991.

He was with the Central Research Laboratories of Thomson-CSF for three years, working on problems of infrared image segmentation using mathematical morphology. He then joined a start-up company, Animation Science, in 1995, as Director of Research and Development. In 1998, he joined OCÉ Print Logic Technologies as a Senior Scientist, where he worked on various problem of image analysis dedicated to scanning and printing. Since 2002, he has been an Associate Professor with the A2SI Laboratory, ESIEE, Paris, and a member of the Institut Gaspard Monge.

His current research interest is in discrete mathematical morphology.

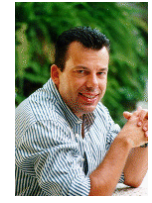

Jacques Facon received the Ph.D. degree in System Control from the UTC Université de Technologie, Compiègne, France, in 1987. He was a Visiting Professor at the Departamento de Engenharia Elétrica e Informática Industrial, Universidade Tecnológica Federal do Paraná, Curitiba, Brazil, 1988-1997, an Associate Professor at the Departamento de Informática, Pontifícia Universidade Católica do Paraná, Curitiba, Brazil, 1997-2003. Since 2003, he has been a Full Professor at the Programa de Pós-Graduação em Informática Aplicada, Pontifícia Universidade Católica do Paraná, Curitiba, Brazil.

His research interests include digital image processing, mathematical morphology and computer vision applications.

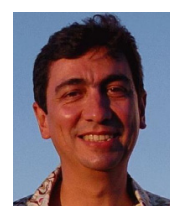

Arnaldo de A. Araújo received his B.Sc., M.Sc. and D.Sc. degrees in Electrical Engineering, from the Universidade Federal da Paraiba (UFPB), Brazil, in 1978, 1981 and 1987, respectively. Arnaldo is currently an Associate Professor at the Departamento de Ciência da Computação (DCC), Universidade Federal de Minas Gerais (UFMG), Belo Horizonte-MG, Brazil (since 1990). He was a Visiting Researcher at the Department d'Informatique, Groupe ESIEE Paris, France, 1994-1995, an associate professor at the Departamento de Engenharia Elétrica, UFPB, 1985-1989, a Research Assistant at the Rogowski-Institut, Technische Hochschule Aachen, Germany, 1981-1985, and an Assistant Professor at DEE/UFPB, 1978-1985. He is the header of the Núcleo de Processamento Digital em Imagens and coordinator of the international cooperation agreement between UFMG and the French Schools ESIEE Paris and ENSEA Cergy-Pontoise.

His research interests include digital image processing and computer vision applications to medicine, fine arts, and content based information retrieval. 